\title{
Effects of fuel properties on the burning characteristics of collision-merged alkane/water droplets
}

\author{
C.H. Wang ${ }^{\mathrm{a}, *}$, K.L. Pan ${ }^{\mathrm{a}}$, W.C. Huang ${ }^{\text {a }}$, H.C. Wen ${ }^{\mathrm{a}}$, J.Y. Yang ${ }^{\mathrm{b}}$, C.K. Law ${ }^{\mathrm{c}}$ \\ ${ }^{a}$ Department of Mechanical Engineering, National Taiwan University, Taipei 106, Taiwan, ROC \\ ${ }^{\mathrm{b}}$ Institute of Applied Mechanics, National Taiwan University, Taipei 106, Taiwan, ROC \\ ${ }^{\mathrm{c}}$ Department of Mechanical and Aerospace Engineering, Princeton University, Princeton, NJ 08544, USA
}

Received 8 November 2005; received in revised form 26 November 2007; accepted 26 November 2007

\begin{abstract}
The combustion characteristics of freely falling droplets, individually generated by the merging of colliding alkane and water droplets, were experimentally investigated. The outcome of the collision droplets was firstly studied and then the subsequent burning processes such as the flame appearance, ignition and burning behaviors were recorded, through either visual observation or microphotography with the aid of stroboscopic lightening. If the merged droplets were exhibited in an insertive manner, while the water droplet inserted into the alkane droplet, these yield the burning behaviors prior to the end of flame were very much similar to that of pure alkane. The burning was ended with droplet extinction for lower-C alkane, and with either droplet "flash vaporization" or extinction for hexadecane. And if the merged droplets were in adhesive manner, for hexadecane with large water content, they either could not be ignited for the large merged droplets, or be ignited with a much prolonged ignition delay, followed by a soot-reducing flame and an ending of droplet extinction for the small merged droplets. "Homogeneous" explosion was not observed in any of the tests, and "heterogeneous" explosion, induced by trapped air bubbles, occasionally occurred for merged droplets with C-atom in alkane is higher than dodecane. And the sudden disappearance of droplet definitely decreased the burning time and thus enhanced the burning intensity. Besides, the fuel mass consumption rates were increased, even in the cases that having droplet extinction, because of the enlargement of the surface area due to the stuffing of water droplet.
\end{abstract}

Published by Elsevier Inc.

Keywords: Collision-merged droplet; Flash vaporization; Extinction

\section{Introduction}

The performance of burners such as diesel engines, furnaces, and gas turbines that utilize high-boiling-point liquid fuels can be adversely affected by the heterogeneous nature of the combustion process, which may result in deleterious outcomes such as high emission levels of $\mathrm{NO}_{x}$ and particulates. The possibility of using water to modify the performance of these devices has been extensively investigated since Hopkinson [1] sprayed water into the combustion chamber. Since water and oil do not mix, in these

\footnotetext{
${ }^{*}$ Corresponding author. Tel.: +8862 33662728; fax: +886223631755. E-mail address: chinghua@ntu.edu.tw (C.H. Wang).
}

studies they were either injected separately into the combustion chamber, or first emulsified before injection. Direct water injection can either lower the combustion temperature and thus the emission of $\mathrm{NO}_{x}$, or increase the fuel mass flux, for a given chamber temperature, and thus the overall power output. For emulsions, studies have shown the possibility of enhanced fuel efficiency and reduced particulate emission [2-8].

Fundamental studies on the combustion of water/oil emulsion droplets were initiated by Ivanov and Nefedov [9], followed by Lasheras et al. [10], Wang and Law [11], and Wang and Chen [12]. Results showed that the droplets could either spontaneously and/or consecutively explode during combustion. It was thus suggested that this socalled microexplosion event was responsible for the 
observed faster burning rates and reduced particulate emissions. A comprehensive model was formulated by Law [13] to describe the gasification mechanism of emulsion droplets, with a criterion governing the onset of microexplosion.

Recently Wang et al. [14] reported that a single entity consisting of the nominally immiscible masses of oil and water was frequently formed by colliding an oil droplet with a water droplet. The burning characteristics of such collision-generated water/hexadecane droplets were subsequently studied in detail. Various positive effects similar to those of emulsification were observed, particularly those associated with microexplosion. It was also shown that the merged mass could take the form of either adhesion, for which the individual masses of hexadecane and water seemed to attach to each other with a distinctive interface, or insertion, for which the water droplet penetrated into and was thereby engulfed by the hexadecane droplet.

In view of the potential usefulness of such collisionmerged oil/water droplets for practical applications, and recognizing that the associated combustion response must necessarily depend on the volatility and rheology of the oil component, we have extended the previous study on hexadecane to several other alkanes spanning over an extensive range of volatilities. It will be shown in due course that substantial difference in the combustion behavior indeed exists for these oil components of different properties.

In the next section the experimental aspects of the investigation is specified, which will be followed by the presentation and discussion of the experimental results.

\section{Experimental apparatus and procedure}

The experimental apparatus and procedure were described in Wang et al. $[14,15]$ and hence are only briefly discussed here. Specifically, two independently controlled droplet streams with identical droplet generation rate were produced using a modified ink-jet printing technique, and were made to collide in the desired manner by manipulating the phase difference and/or the relative positions of the two generators. The collided droplets then fell through a small vertical connecting channel into a burner-generated, high-temperature, oxidizing environment in which the droplets ignited and burned. The environment had an oxygen concentration of $23.5 \mathrm{vol} . \%$ and a maximum temperature of $1030{ }^{\circ} \mathrm{C}$, which was subsequently reduced by approximately $18^{\circ} \mathrm{C} / \mathrm{cm}$ along the droplet trajectory. The droplets entered the chamber with a velocity of about $70 \mathrm{~cm} / \mathrm{s}$, with an estimated droplet Reynolds number of $\mathrm{O}(1)$.

The droplet generation frequency was set at $10 \mathrm{~Hz}$ so that the droplets were separated by more than several hundreds of diameters in order to minimize their interaction. A strobe light synchronized with the droplet generators, with variable phase lag and $\pm 0.04 \mathrm{~ms}$ time resolution, was used to freeze the moving droplets at various stages of their lifetime. Enlarged droplet images were recorded by a CCD camera with a Bausch \& Lomb Mono-zoom-7 long focus microscopic lens. The uncertainty in the determination of the droplet size was estimated to be about $2 \%$, mainly from reading the boundary of the droplet image.

The droplet size was varied by altering the size of the glass nozzle. The experiments were conducted with: (1) fixed alkane droplet size at $\sim 280 \mu \mathrm{m}$ and varied water droplet size; (2) fixed water droplet size at $\sim 280 \mu \mathrm{m}$ and varied alkane droplet size; or (3) fixed size of the final merged droplet, at $\sim 320 \mu \mathrm{m}$. These yielded a wide range of water fractions for the collision-merged droplets. To account for the effects of the droplet size on the burning characteristics, data of pure alkane droplets with the corresponding diameters were also obtained for comparison. Technical grade octadecane, hexadecane, dodecane, decane and heptane with purity greater than $99 \%$, and purified water were used in the tests.

In the study, the droplet burning characteristics such as the flame appearance, ignition delay, burning time and rate, and the state of extinction or microexplosion were investigated. The ignition delay was defined as the time interval from the instant at which the droplet was first exposed to the high temperature environment to when a visible flame was observed. The uncertainty in defining each of these instances was $\sim \pm 0.5 \mathrm{~mm}$, and thus the uncertainty of ignition delay was estimated to be $\sim \pm 1 \mathrm{~ms}$. The droplet burning process was assessed through visual observation of the flame streak and microphotography of the droplet image.

It is noted that because of the repeatability and stability of the droplet stream, the phenomena reported in the following actually represent thousands of test droplets at the same condition.

\section{Results and discussion}

\subsection{Collision outcomes}

As noted previously [14], the collision of an oil droplet and a hexadecane droplet frequently results in a single entity with two different structures, namely insertive and adhesive. Fig. 1a and $\mathrm{b}$ demonstrate these two modes of formation. It is seen that, for insertive merging, a spherical mass is formed with hexadecane enclosing the water droplet. For adhesive merging, the two colliding droplets are in contact with each other, exhibiting an irregular shape. It was also found that, as the droplet temperature increases after the droplet enters the hot environment, the adhesive structure would change into the insertive structure, which would be the dominant configuration during burning.

With great care of the photography and extension of the test range, new phenomena were observed on the collision of water and alkane droplets. First, for water/hexadecane collision, it was found that the adhesive structure may not change to that of insertion if the fraction (volume) of water is sufficiently high, even at elevated temperatures. 


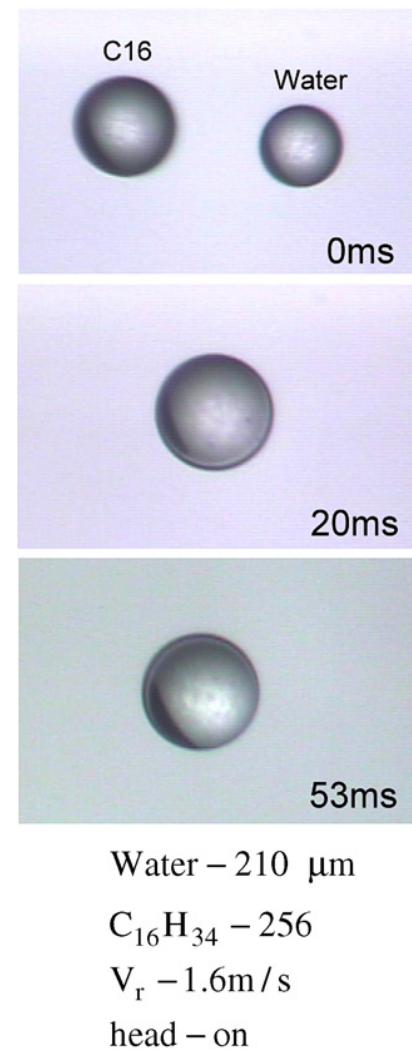

(a) insertive
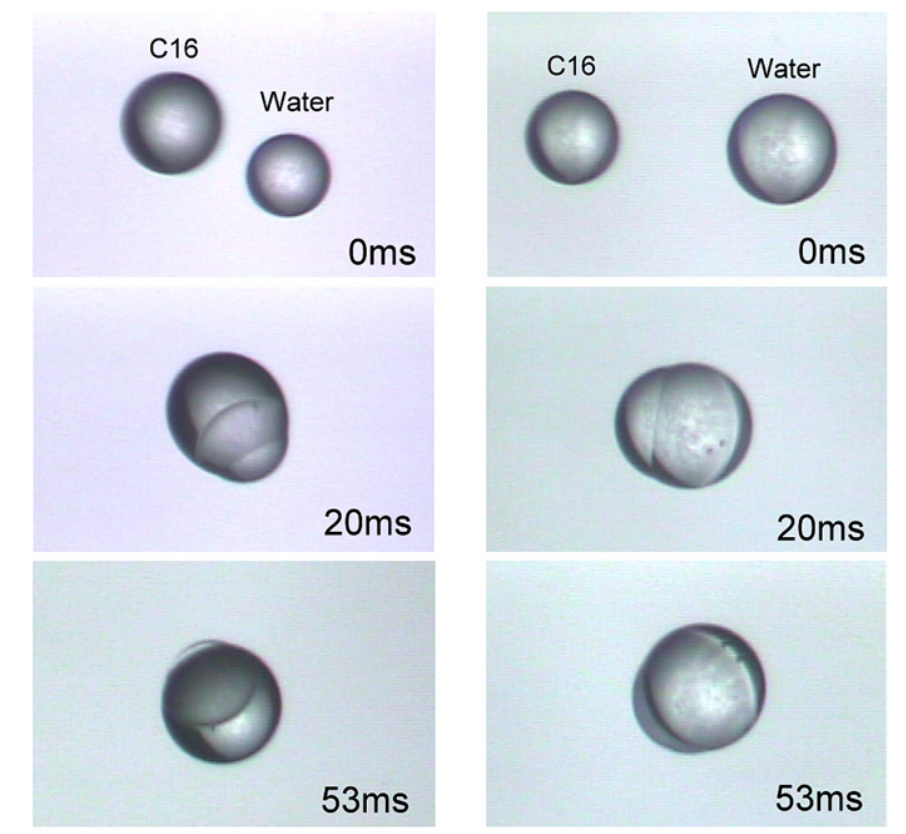

Water $-210 \mu \mathrm{m}$

$\mathrm{C}_{16} \mathrm{H}_{34}-256$

$\mathrm{V}_{\mathrm{r}}-1.6 \mathrm{~m} / \mathrm{s}$

off - center

(b) adhesive

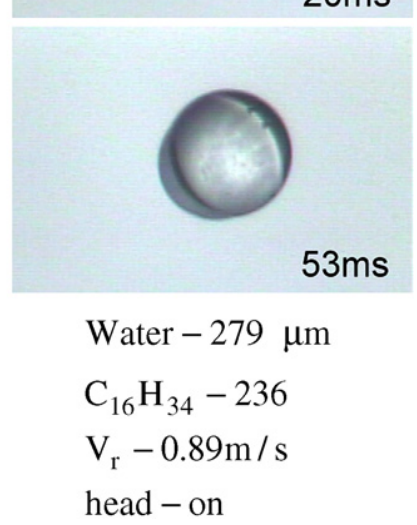

(c) adhesive

Fig. 1. Collision-merged hexadecane/water droplets.

In such cases the enclosed water droplet is always partially exposed to the environment, as shown in Fig. 1c. Its occurrence depends on the relative volume and the absolute sizes of the water and alkane droplets. For example, for merged droplets that have the same water fraction, the one with the smaller water droplet has the thinner alkane surface layer, is easier to have its surface broken and the water droplet exposed. Furthermore, having a higher surface tension, the merged water/hexadecane droplet is more prone to have the water droplet exposed than the water/dodecane or water/heptane droplets. Exposure of the water droplet would then substantially change the burning characteristics of the merged droplet, as will be discussed in the following sections. The second point to note is that the enclosed water droplet in the insertive mode is neither located at the center of the merged droplet nor spherical in shape for the first $\sim 100 \mathrm{~ms}$ of the collision period. It takes about $200-300 \mathrm{~ms}$ to become stable. With increasing water fraction, the enclosed water droplet can be centered and spheroidized more easily. Such differences can lead to different explosion mechanisms.

Similar to that reported in Wang et al. [14], air bubbles were occasionally observed during collision. The trapped air bubbles within the coalescent droplets could play an essential role in inducing early microexplosion for some of the merged water/alkane droplets in the present study.

\subsection{Flame characteristics}

For pure alkanes, the droplet flame streak was blue for a short distance from the ignition location, subsequently becoming white-yellow inside and blue outside for a large portion of the burning trajectory, and turned to blue again as the droplet became small. A yellow sparkle was occasionally observed at the very end, which was likely caused by either the minor impurities originally present in the fuel stock, or the absorption and subsequent concentration of the combustion intermediates during burning. Similar flame behaviors were observed for different droplet sizes, with longer flame lengths for larger droplet sizes.

For water/hexadecane droplets, tests were first conducted by fixing the hexadecane droplet size at $\sim 280 \mu \mathrm{m}$ and increasing the water droplet size from a small value of $\sim 80 \mu \mathrm{m}$, in the same manner as that of Wang et al. [14]. This is denoted as the low-water-fraction (LWF) approach. As such, the corresponding volume fraction of water is increased with increasing size of the merged droplet. With water fraction up to $\sim 0.65$, the appearance of the flame streak prior to flame termination was similar to that of pure hexadecane, except it was slightly dimmer. With further increase the water fraction, the merged droplet could no longer be ignited. Fig. 2a and $\mathrm{b}$ compare the flame images of pure hexadecane and a merged droplet with water fraction of $\sim 0.6$. 


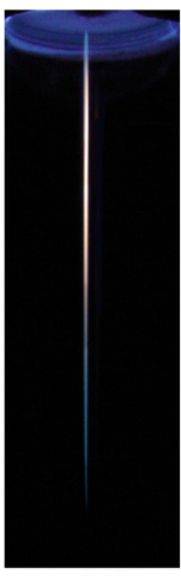

(a)

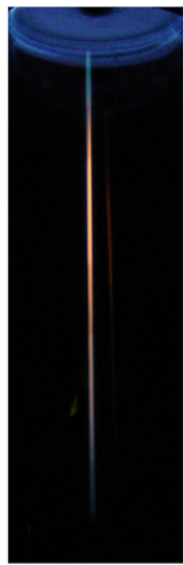

(b)

280 $\mathrm{C}_{16} \mathrm{H}_{34}$

Water

Water fraction 0

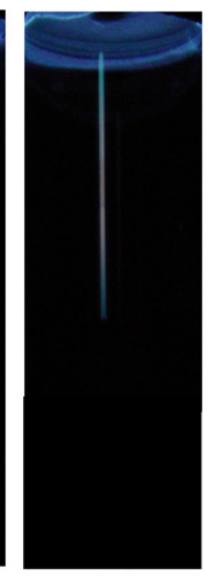

(c)

$233 \mu \mathrm{m}$

$280 \mu \mathrm{m}$

0.63
Fig. 2. Flame photos of merged hexadecane/water droplets.

We have in addition conducted tests by fixing the water droplet size at $\sim 280 \mu \mathrm{m}$ and increasing alkane droplet size from a small value of $\sim 160 \mu \mathrm{m}$. This is referred to as the high-water-fraction (HWF) approach. It allows the merged droplet to have very high water fraction but relatively small size. It was found that the merged droplet could still be ignited even with water fraction up to $\sim 0.84$, while the overall flame streak was blue and almost invisible. Fig. 2c shows the flame images with water fraction of $\sim 0.63$ with this approach. The difference in the flame appearance between Fig. 2b and $c$ is due to the fact that burning in the LWF approach is dominated by that of hexadecane, with the enclosed water droplet simply serving as a heat sink. However, in the HWF approach substantial water is vaporized because the water droplet is exposed. This leads to a larger expenditure for the vaporization heat as well as a higher water concentration in the flame, both of which lead to a lower flame temperature and hence luminosity.

For water/dodecane and water/heptane droplets, with fixed fuel droplet size at $\sim 280 \mu \mathrm{m}$ and increased water droplet size, ignition could still be accomplished with water fractions as high as 0.7 or even higher. The difference between the two fuels is that as the water fraction was increased to $\sim 0.7$ for the water/dodecane droplet, the inserted water droplet might be exposed, leading to weakening of the flame luminosity. For the water/heptane droplet, the flame was still as bright as that of pure heptane even for water fraction up to 0.7 , indicating the submerged nature of the water droplet.

After the steady burning period, the flame streaks terminated either with droplet explosion, via which the droplet disintegrates into mists or pieces, or with flame extinction, via which a droplet still exists after the disappearance of the flame. In both cases, the appearances of the flame prior to termination were quite similar, with gradual disappearance of the faint-blue luminosity. However, for the explosion cases, the flame tip was flattened and one could continuously hear violently explosive noise.

For water/hexadecane droplets, with the LWF approach the flame terminated with extinction for low water fractions, say $<0.11$, while it terminated with droplet explosion for water fractions between 0.11 and 0.65 . With the HWF approach, burning was still possible even with water fractions as high as $\sim 0.84$. Burning was terminated with flame extinction for water fractions higher than $\sim 0.65$, and with droplet explosion for lower values. For water/dodecane and water/heptane droplets, all burning were terminated with flame extinction.

The flame characteristics of the merged water/octadecane droplets were also investigated by fixing the final merged droplet size at $\sim 320 \mu \mathrm{m}$, with water fractions of $\sim 0.25,0.5$ and 0.75 . The appearance of the flame for water fractions of $\sim 0.25$ and 0.5 was similar to that for the water/ hexadecane droplet, with both ending with a flattened tip indicating the occurrence of droplet explosion. With the higher water fraction of $\sim 0.75$, the flame streak had a long, faint blue tail, indicating exposure of the water droplet, and terminated with extinction.

It is noted that there was no visible light emitted during the explosion of water/hexadecane and water/octadecane, indicating that there was not much fuel left in the disintegrating droplet. This will be further discussed in relation to the extinction and microexplosion of droplets.

Early microexplosion was observed by Wang et al. [14] for the collision-merged water/hexadecane droplets, which was called "heterogeneous" nucleation. It was caused by air bubbles trapped within the merged droplet and resulted in a branched flame. The phenomenon was occasionally observed in this study, and it also occurred for the merged water/dodecane droplets.

\subsection{Ignition delays}

Fig. 3 shows the ignition delays $\left(t_{\mathrm{ig}}\right)$ of the merged water/hexadecane droplets obtained by the two different approaches. The final merged droplet size varied from $\sim 280$ to $400 \mu \mathrm{m}$ such that the corresponding water fraction varied from 0 to 0.65 for the LWF approach and $0.84-0.36$ for the HWF approach. The ignition delay for the pure hexadecane droplet with the corresponding size was also determined for comparison. It increased with increasing droplet size, as expected [16].

For investigations using the LWF approach, since the water droplet was embedded within the merged mass, hexadecane was the only component that was vaporized and as such there was no chemical difference in the ignition mechanism between the merged and pure hexadecane droplets. Consequently the slight increase in the ignition delay for the merged droplet was caused by the higher heat capacity of water, which slowed the increase in the droplet temperature. When the water fraction of the merged droplet 


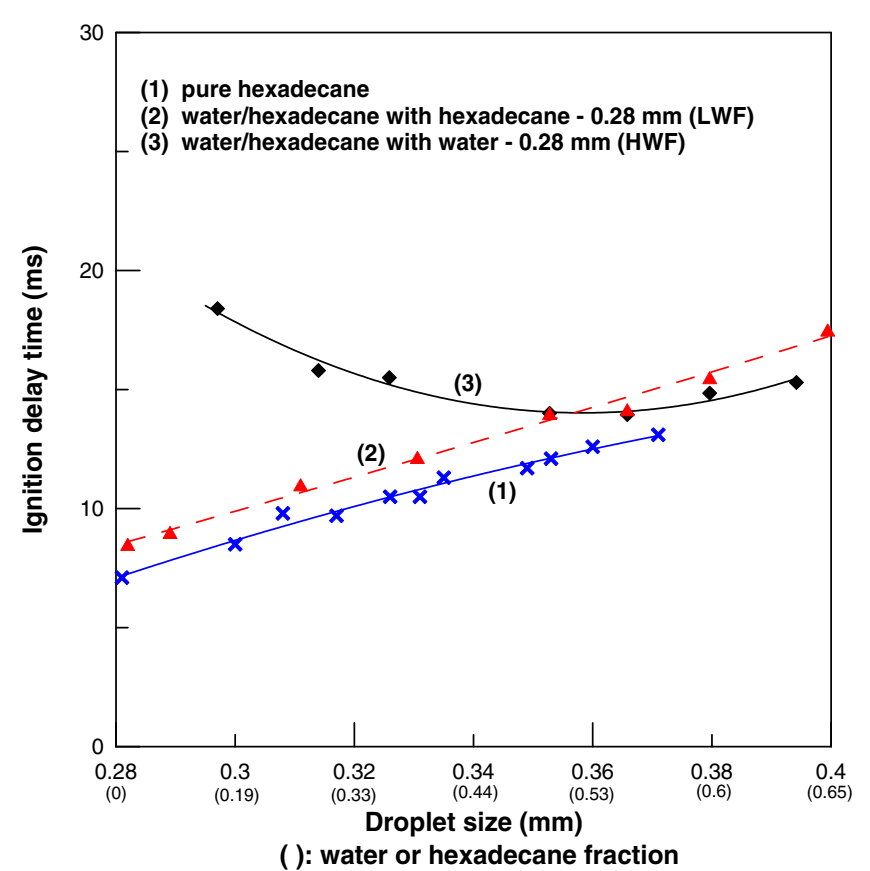

Fig. 3. Ignition delays of merged hexadecane/water droplets.

became close to 0.65 , it could no longer be ignited because of the exposure of the water droplet.

With the HWF approach, the merged droplet could still be ignited even as the water fraction was up to 0.84 , but with a substantially prolonged ignition delay. Recognizing that the water droplet was exposed for water fraction higher than $\sim 0.65$, the result that the ignition delay decreased with decreasing water fraction for water fraction from $\sim 0.84$ to 0.65 indicates that the increase in the hexadecane concentration dominated over the increase of the droplet size. As the water fraction decreased, the water droplet became completely enclosed, and the effect of decreasing water fraction was gradually offset by the increased droplet size. The trend of ignition delay then leveled off and started to increase. With further increase of the hexadecane content, the ignition delay approached that of pure hexadecane.

The variation of ignitability when the water droplet was exposed merits additional consideration. Specifically, the temperature of the merged droplet started to increase when the droplet was exposed to the high temperature environment. However, the temperature of the droplet surface does not follow the Raoult's Law for liquid mixtures [13] because water and oil do not mix. Consequently, the oil and water components will each dictate its own surface temperature and the associated vapor concentration, resulting in a temperature distribution over the droplet surface. Since the boiling point of hexadecane is substantially higher than that of water, it is then reasonable to expect that the surface temperature on the water side is close to the boiling point of water while that on the fuel side should also be high enough in order to have the fuel vapor to achieve ignition and sustain burning. However, the high heat capacity and latent heat of water slowed the tempera- ture increase on the water surface because of the heat transfer from hexadecane to water, and thereby the temperature increase of hexadecane. Thus, the ignition delay is much prolonged, as shown in Fig. 3.

The ignition delay for a pure dodecane droplet was increased with the increase of its size, similar to hexadecane. For heptane, however, the ignition delay ceased to monotonically increase with the droplet size, and as such exhibited behavior showing that ignition was controlled by chemical reactivity $[17,18]$. For merged water/dodecane and water/heptane droplets, tests were conducted only with the LWF approach. As shown in Fig. 4, within the test range, with water fraction up to $\sim 0.7$, the ignition delay was almost unaffected by the addition of water, indicating the enclosed nature of the water droplet.

\subsection{Extinction and microexplosion}

Depending on the combustion conditions, a weakly burning droplet may self extinguish as its size is reduced to a certain critical value $[19,20]$. For an immiscible bi-component droplet, the burning droplet may explode as one of the components reaches it limit of superheat [13].

All the pure alkane droplets tested achieved complete burning in the present environmental conditions, occasionally accompanied by a tiny sparkling flame at the very end, possibly due to the concentrated impurities.

For collision-merged water/hexadecane droplets, the flame terminated with either flame extinction or droplet explosion. Fig. 5 shows the residual droplet size at flame termination, with data being obtained from both the

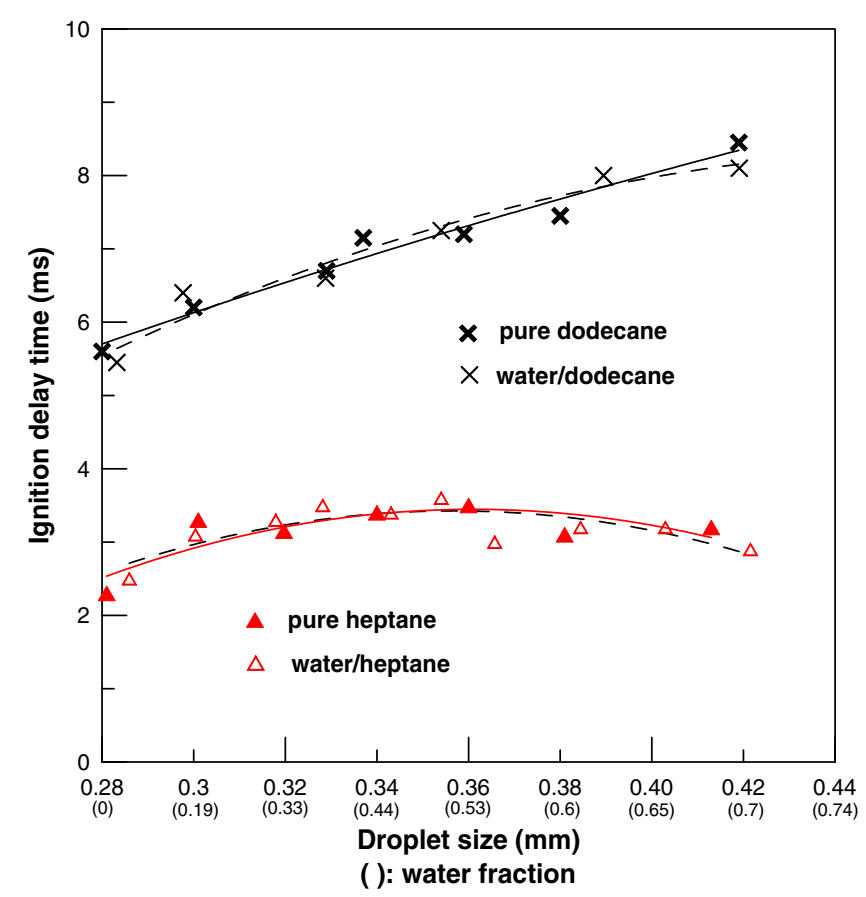

Fig. 4. Ignition delays of merged dodecane/water and heptane/water droplets. 


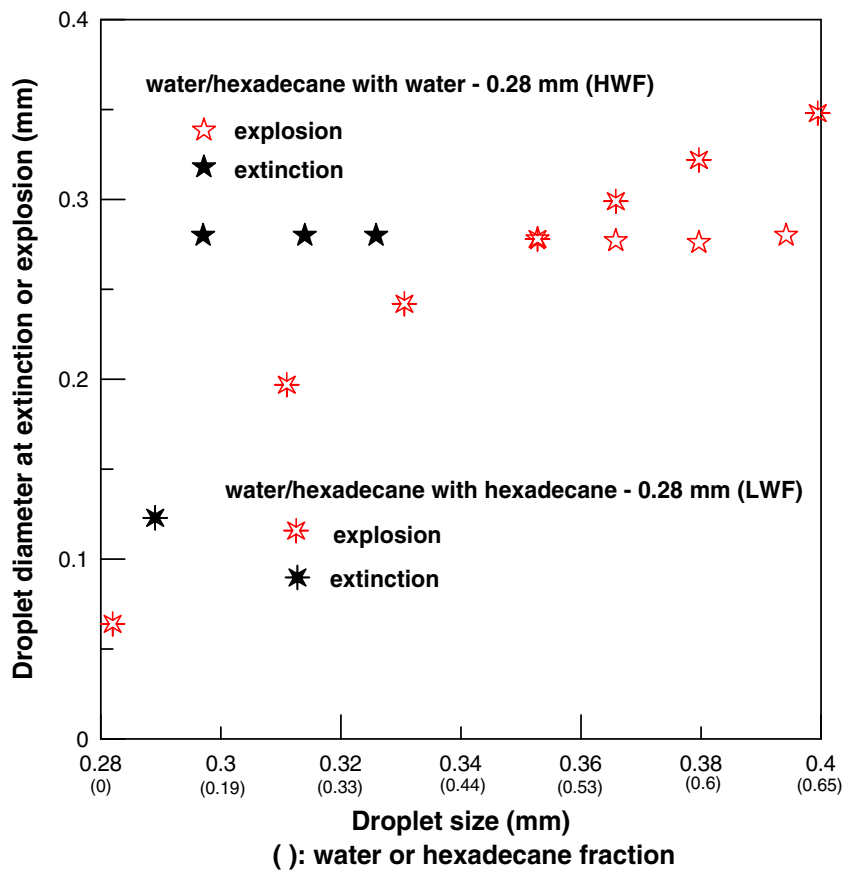

Fig. 5. Residual droplet sizes at flame extinction for merged hexadecane/ water droplets.

LWF and HWF approaches. It is seen that they are all close to the initial size of the colliding water droplet.

Combining the results of flame appearance, invisible flame termination, and residual droplet size, we conclude that a collision-merged droplet with embedded water droplet would burn in the manner of a pure hexadecane droplet until hexadecane is mostly consumed. As the embedded, superheated water droplet was exposed to the ambience, it either simply extinguished or instantly gasified through microexplosion in the manner of flash vaporization. The surface temperature of hexadecane, in any cases, is not high enough to have the droplet undergo explosion through the "homogeneous" nucleation of water [13]. Furthermore, early explosion was occasionally observed and was most likely caused by the trapped air bubble at the interface between water and hexadecane upon droplet collision, but without any consistent trend. The air bubble serves as a "heterogeneous" site that can induce nucleation at much lower temperature, ostensibly before the embedded water droplet is exposed to the ambience, with the burning droplet disintegrating into many small pieces. Fig. 6a and b, respectively show the typical images of droplet explosion for flash vaporization and heterogeneous nucleation.

For those situations with small droplet size, ignition could still be achieved for the merged water/hexadecane droplets with the water droplet exposed to the ambience. However, the exposure limited the temperature of the water surface and subsequently lowers the temperature of hexadecane. As a consequence, the burning was terminated with flame extinction. Furthermore, the residual droplet size did not differ much from the initial size of the water droplet.

It is noted that similar results were also observed for the merged water/octadecane droplets, implying that the sur-

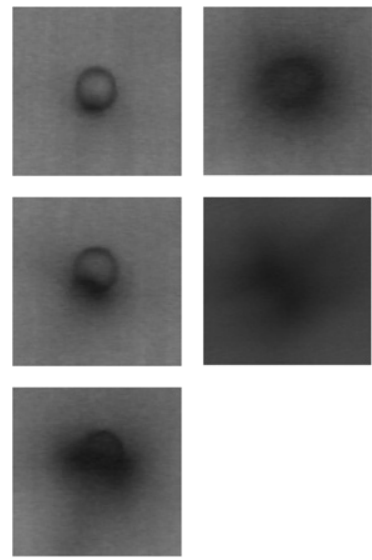

(a) flash vaporization
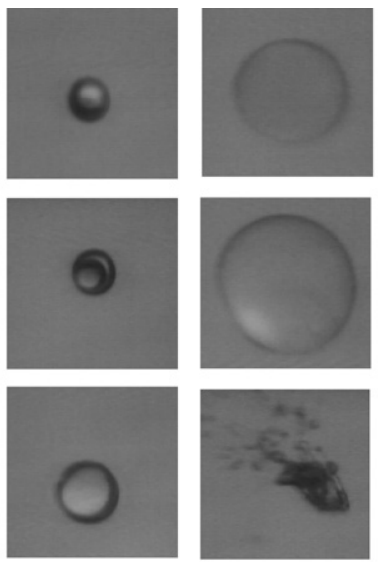

(b) heterogeneous explosion
Fig. 6. Different images of droplet explosions.

face temperature of octadecane was still not high enough to "homogeneously" nucleate the embedded water droplet.

For water/dodecane and water/heptane droplets, the burning terminated with extinction for all water contents because of the lower droplet surface temperature. This is corroborated by Fig. 7, which shows that the residual droplet size at flame termination is close to the initial size of the water droplet. It is nevertheless also noted that "heterogeneous" explosion was occasional observed for merged water/dodecane droplets.

\subsection{Burning rates}

Even with a pure fuel, due to the requirement for droplet heating and the variation of Reynolds number associated

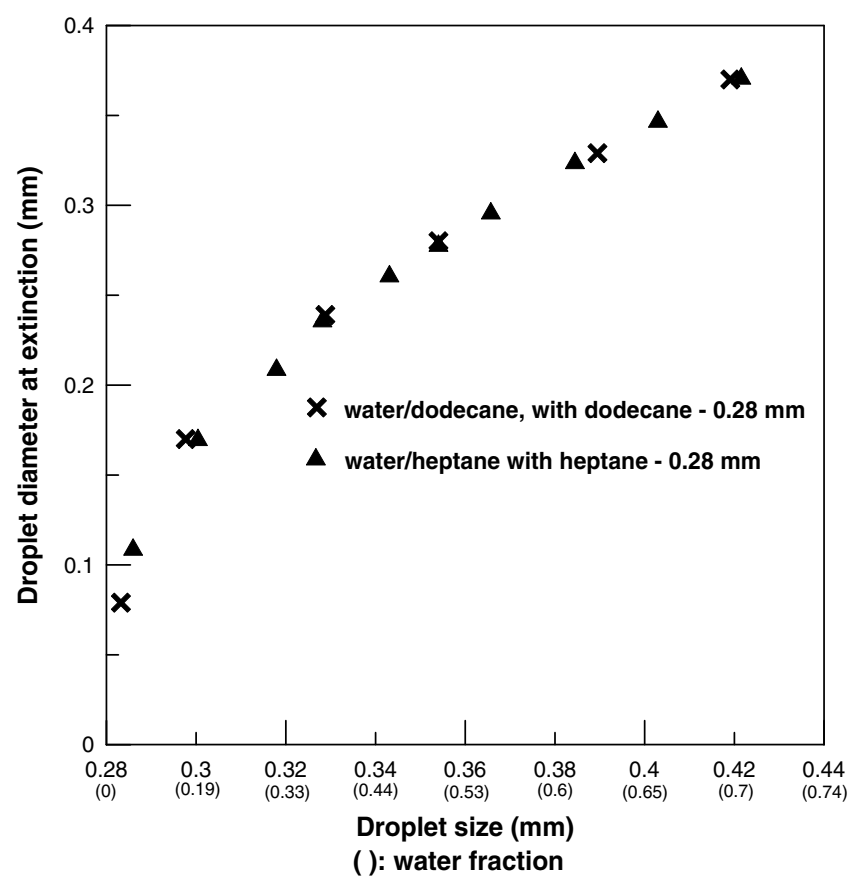

Fig. 7. Residual droplet sizes at flame extinction for merged dodecane/ water and heptane/water droplets. 
with the moving droplet, the temporal variation of the square of the droplet diameter is not as linear as required by the $d^{2}$-law of droplet burning. Furthermore, complex phenomena are present for the collision-merged water/ alkane droplet, such as embedding or exposure of the water droplet, and extinction and microexplosion. Thus it is more informative to define the rate of fuel conversion based on some global experimental parameters that account for the processes of ignition, steady burning, extinction, and microexplosion. These parameters are: initial droplet diameter, $D_{0}$, the extinction and microexplosion droplet diameters, $D_{\text {ext }}$ and $D_{\text {exp }}$, respectively, the burning time of the droplet, $\Delta t_{1}$, which is the time between ignition and either extinction or microexplosion, and the droplet lifetime, $\Delta t_{\mathrm{ol}}$, where $\Delta t_{\mathrm{o} 1}=\Delta t_{1}+t_{\mathrm{ig}}$, which is the time between the instant the droplet is first exposed to the hot ambient and the occurrence of either extinction or microexplosion. We can then define the average, overall and effective burning rates, $K_{\mathrm{ab}}, K_{\mathrm{ob}}$, and $K_{\mathrm{eff}}$ as

$K_{\mathrm{ab}}=\left(D_{0}^{2}-D_{\mathrm{ext}}^{2}\right) / \Delta t_{1}$ and $K_{\mathrm{ob}}=\left(D_{0}^{2}-D_{\mathrm{ext}}^{2}\right) / \Delta t_{\mathrm{ol}}$,

for burning terminated by extinction, and

$$
\begin{aligned}
K_{\mathrm{ab}} & =\left(D_{0}^{2}-D_{\exp }^{2}\right) / \Delta t_{1}, \quad \mathrm{~K}_{\mathrm{ob}}=\left(D_{0}^{2}-D_{\exp }^{2}\right) / \Delta t_{\mathrm{ol}}, \text { and } K_{\text {eff }} \\
& =\left(D_{0}^{2}\right) / \Delta t_{\mathrm{o} 1}
\end{aligned}
$$

for burning terminated by microexplosion or by almost complete burning.

Data of burning times and lifetimes of water/hexadecane droplets are plotted in Fig. 8, which is to be compared with those of water/dodecane and water/heptane as shown in Fig. 9. The results of pure alkanes with different sizes are also shown for comparison. The different burning rates as

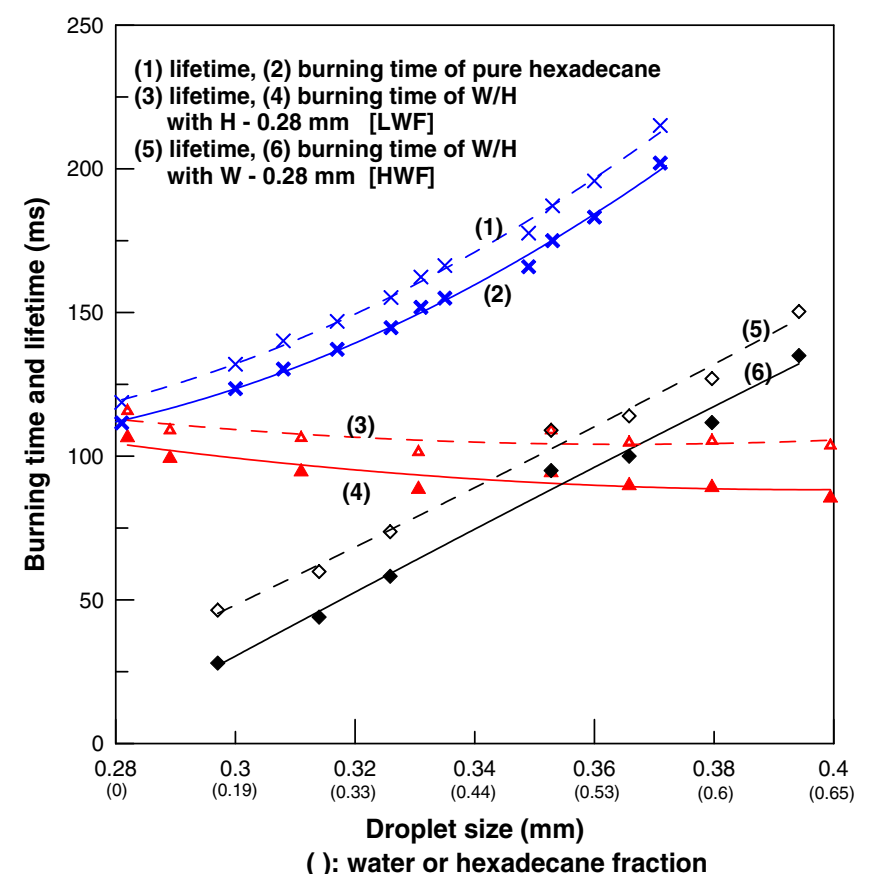

Fig. 8. Burning times and lifetimes of merged and pure hexadecane droplets.

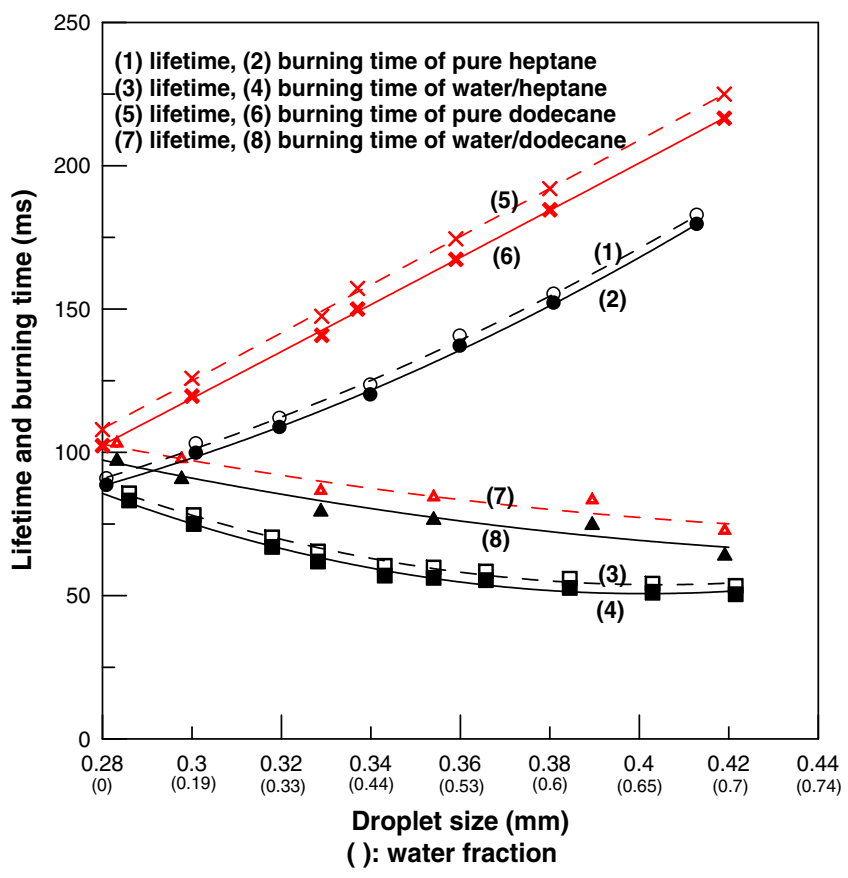

Fig. 9. Burning times and lifetimes of merged and pure dodecane, heptane droplets.

defined can thus be calculated accordingly. For hexadecane and dodecane, since microexplosion induced by air bubbles was only occasionally observed, results from these events are not included in the analysis.

It is seen from Figs. 8 and 9 that the burning times $\Delta t_{1}$ of pure alkanes increased with increasing droplet size, as expected. However, with the same droplet size, because of different outcomes of extinction and microexplosion, the burning times for the merged droplets decrease with increasing water content.

Figs. 10-12 show the burning rates of merged and pure fuel droplets for hexadecane, dodecane, and heptane respectively. For pure alkanes, Fig. 12 reflects the facts that while the burning rate increased with increasing droplet size due to the corresponding increase of the droplet Reynolds number, the trend is offset by the gradually reducing ambient temperature. Furthermore, because of the extra heating needed for the embedded water droplet, the average and overall burning rates decrease with increasing water fraction of the merged droplet.

The effective burning rate is meaningful for droplets that were completely burned out - with or without microexplosion, and is meaningless for droplet burning that terminated with extinction. If microexplosion occurred, the droplet disappeared almost immediately upon explosion, implying that a droplet with given size existed only from the instant it was introduced to the combustion environment until it exploded. Consequently, the effective burning rate represents the most practical description to include the various factors that affected droplet burning. Fig. 13 shows that, with the aid of microexplosion, the effective burning rate did increase with increasing water content. 


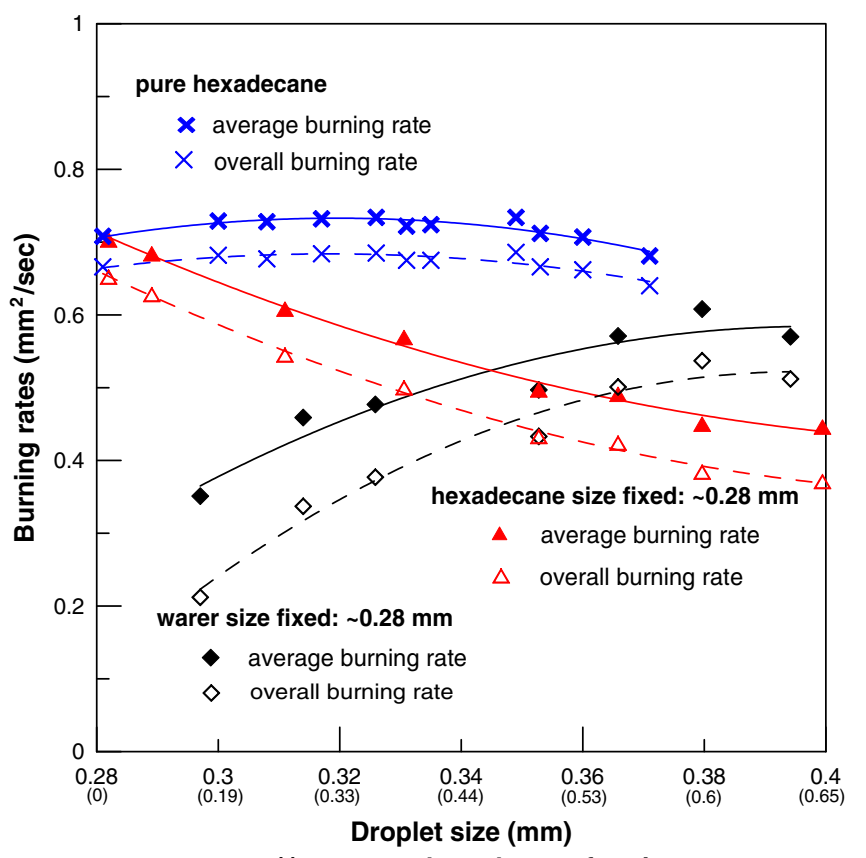

(): water or hexadecane fraction

Fig. 10. Average and overall burning rates of merged and pure hexadecane droplets.

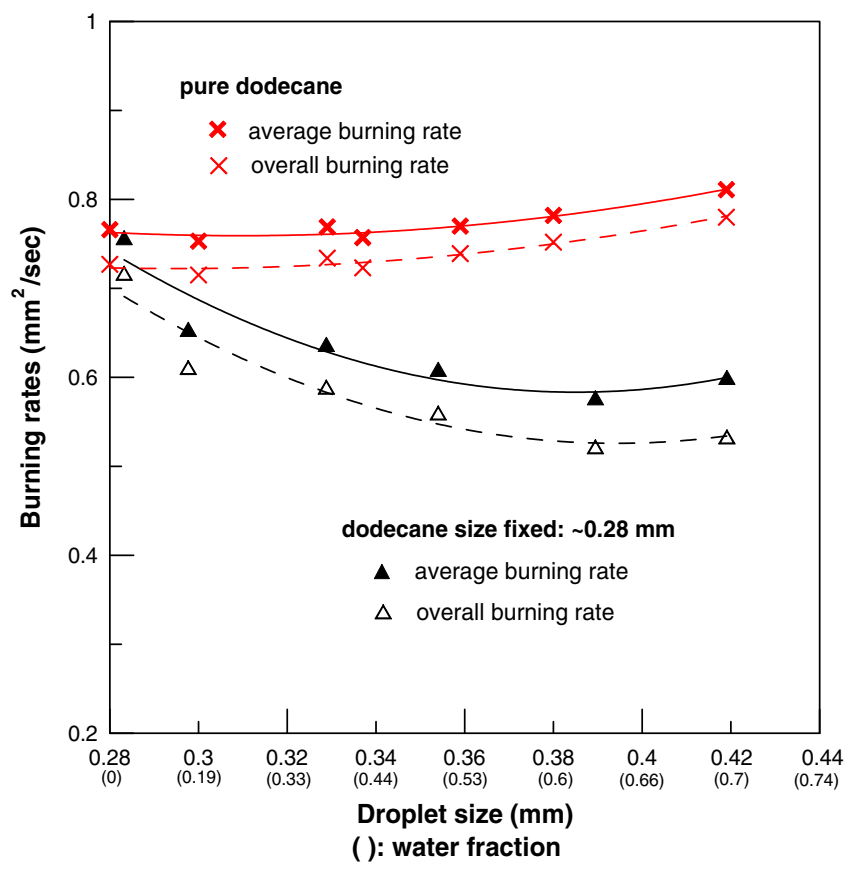

Fig. 11. Average and overall burning rates of merged and pure dodecane droplets.

Without microexplosion, one might intuitively expect that adding water has only deteriorating effect on droplet combustion. It is, however, also instructive to consider the efficiency of burning from the viewpoint of a fuel consumption rate-defined as $\dot{m}_{\text {fuel }}=\mathrm{d} m_{\text {fuel }} / \mathrm{d} t$, or, for simplic-

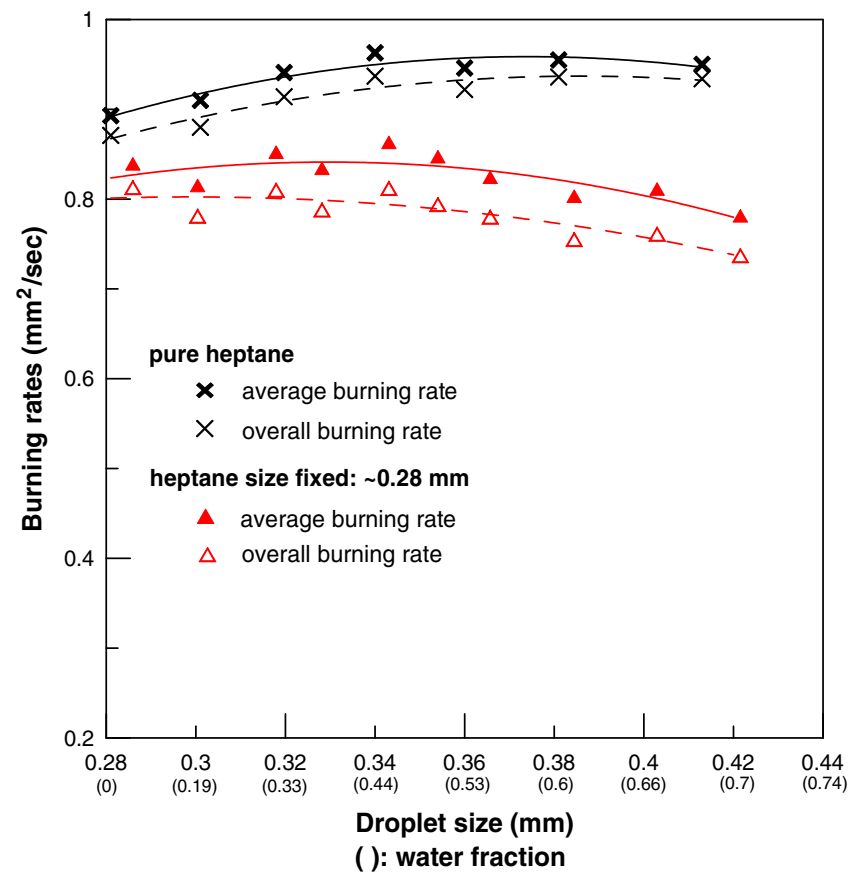

Fig. 12. Average and overall burning rates of merged and pure heptane droplets.

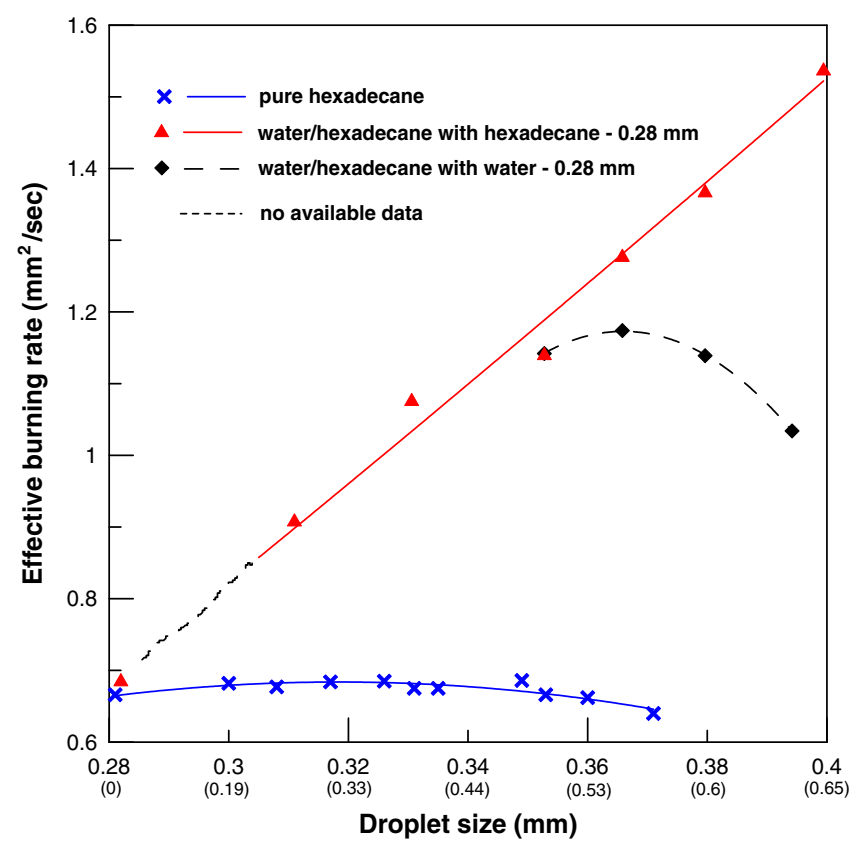

( ): water or hexadecane fraction

Fig. 13. Effective burning rates of merged and pure hexadecane droplets.

ity, a global or averaged fuel consumption rate $\dot{\bar{m}}_{\text {fuel }}=m_{\text {fuel }} / \Delta t_{\mathrm{ol}}$. Fig. 14 plots the "normalized" result, that is, the ratio of the fuel consumption rate of the merged droplet with fixed alkane size at $\sim 280 \mu \mathrm{m}$ to that of pure alkane with a size of $\sim 280 \mu \mathrm{m}$. The results show that the 


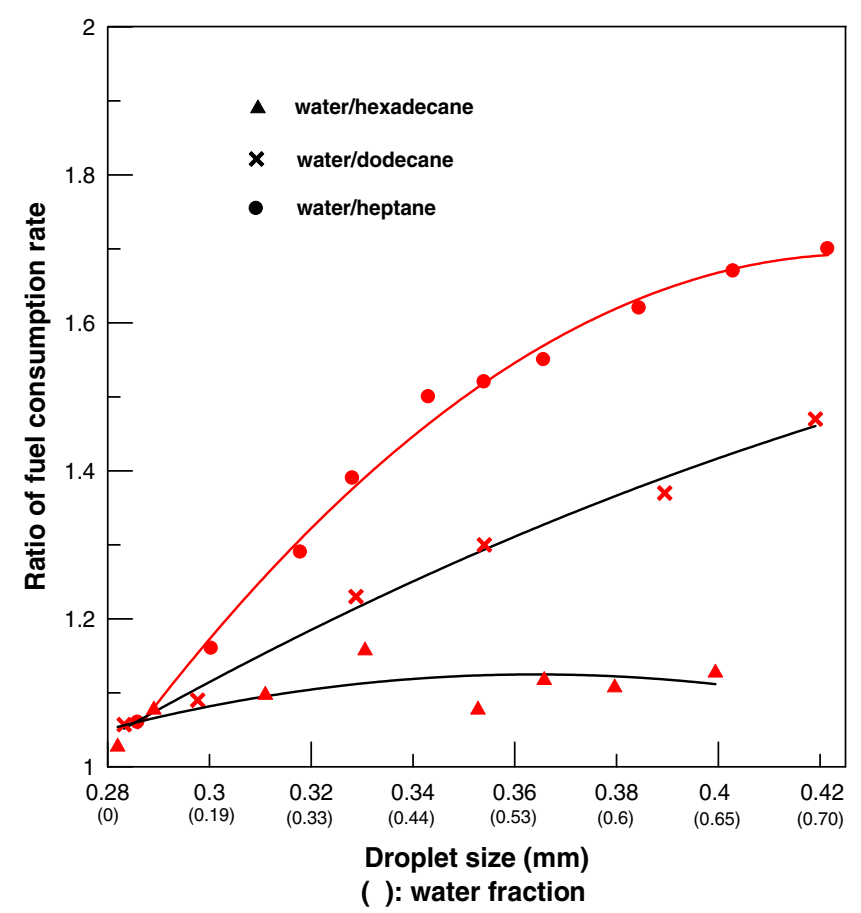

Fig. 14. Ratios of fuel mass consumption rate of merged alkane droplets.

normalized global fuel consumption rate always increased with increasing water content, and that adding water in heptane was more effective than adding it into dodecane and hexadecane. It is quite clear that during the burning period, $\dot{m}_{\text {fuel }} \propto\left(\mathrm{d} D^{3} / \mathrm{d} t\right) \propto D K$. Thus the fuel consumption rate can be significantly increased with a larger droplet size $D$ but slightly reduced burning rate $K$ for water-embedded droplets.

\section{Conclusions}

Results and understanding gained from the present study are summarized in the following.

1. Droplets of two immiscible liquids, water and alkane in the present study, merge upon collision with either adhesive or insertive modes. With increasing droplet temperature upon gasification, an originally adhesively merged droplet could change to an insertive one, except for water/hexadecane droplets with sufficiently large water content, $\sim 0.65$, and/or small water size.

2. For merged water/alkane droplets in insertive manner, with water droplet enclosed, the flame appearance prior to extinction or microexplosion is similar to that of pure alkane. For merged droplets stayed in adhesive manner, with water droplet exposed to the ambient, the flame color changes dramatically from alkane flame, because water and alkane vaporized simultaneously.

3. Compared to pure alkanes, the ignition delay of the merged droplet with enclosed water droplet is slightly longer. As the water droplet is exposed to the ambient, ignition is either suppressed or the ignition delay sharply increased.
4. Homogenous nucleation of the water droplet was not observed in the present tests. Flash vaporization, involving the sudden gasification of the enclosed and superheated water droplet as it exposed to the ambience, was observed for some of the merged water/octadecane and water/hexadecane droplets. Heterogeneous nucleation, induced by trapped air bubbles, was occasionally observed for merged droplets of octadecane, hexadecane, and dodecane droplets. Extinction of the merged droplet was observed for the rest of tests.

5. Average and overall burning rates for merged water/ alkane droplets all decrease with water addition. However, if microexplosion occurred, the effective burning rate was increased, from $\sim 0.7 \mathrm{~mm}^{2} / \mathrm{sec}$ to $\sim 1.5 \mathrm{~mm}^{2} /$ sec.

6. The net fuel consumption rate increased with the water addition, with the increasing in the order of heptane $>$ dodecane $>$ hexadecane.

\section{Acknowledgements}

The work at National Taiwan University was supported by the National Science Council of Taiwan, ROC, and that at Princeton University was supported by the US Air Force Office of Scientific Research.

\section{References}

[1] B.P. Hopkinson, in: Proceedings of the Institute of Mechanical Engineering, 1913, p. 679.

[2] I. Cornet, W.E. Nero, Emulsified fuels in compression ignition engines, Industry and Engineering Chemistry 47 (1955) 21332141.

[3] E. Valdmanis, D.E. Wulfhorst, The Effect of Emulsified Fuels and Water Induction on Diesel Combustion, SAE 700736, 1970.

[4] G. Greeves, I.M. Khan, G. Onion, Effects of water induction on diesel engine combustion and emissions, in: 16th International Symposium on Combustion, 1976, pp. 321-336.

[5] T. Murayama, Experimental reduction of $\mathrm{NO}_{x}$, Smoke and BSFC in a Diesel Engine Using Uniquely Produced Water to Fuel Emulsion, SAE 780224, 1978.

[6] C.H. Wang, L.H. Ni, The feasibility study of water-oil emulsion in diesel engine, The Chinese Journal of Mechanics 12 (4) (1996) 465474.

[7] C.Y. Lin, K.H. Wang, Diesel engine performance and emission characteristics using three-phase emulsions as fuel, Fuel 83 (2005) $537-545$.

[8] C.Y. Lin, L.W. Chen, Engine performance and emission characteristics of three-phase diesel emulsions prepared by an ultrasonic emulsification method, Fuel 85 (2006) 593-600.

[9] V.M. Ivanov, P.I. Nefedov, Experimental Investigation of the Combustion Process of Natural and Emulsified Liquid Fuels, NASA Tech. Translation TTF-258, 1965.

[10] J.C. Lasheras, A.C. Fernandez-Pello, F.L. Dryer, Initial observations on the free droplet combustion characteristics of water-in-fuel emulsions, Combustion Science and Technology 21 (1979) 1-14.

[11] C.H. Wang, C.K. Law, Microexplosion of fuel droplets under high pressure, Combustion and Flame 59 (1985) 53-62.

[12] C.H. Wang, J.T. Chen, An experimental investigation of the burning characteristics of water-oil emulsions, International Communication of Heat Mass Transfer 23 (6) (1996) 823-834. 
[13] C.K. Law, A model for the combustion of oil/water emulsion droplets, Combustion Science and Technology 17 (1977) 29-38.

[14] C.H. Wang, C.Z. Lin, W.G. Hung, W.C. Huang, C.K. Law, On the burning characteristics of collision-generated water/hexadecane droplets, Combustion Science and Technology 176 (2004) 71-93.

[15] C.H. Wang, X.Q. Liu, C.K. Law, Combustion and microexplosion of freely falling multicomponent droplets microexplosion of fuel droplets under high pressure, Combustion and Flame 56 (1984) 175-197.

[16] C.K. Law, Theory of thermal ignition in fuel droplet burning, Combustion and Flame 31 (1978) 285-296.
[17] M. Takei, T. Tsukamoto, T. Niioka, Ignition of blended-fuel droplet in high temperature atmosphere, Combustion and Flame 93 (1993) $149-156$.

[18] C.H. Wang, D.S. Liou, An experimental investigation of the ignition delay of freely-falling fuel droplets, Journal of the Chinese Society of Mechanical Engineers 17 (4) (1996) 387-396.

[19] C.K. Law, F.A. Williams, Kinetics and convection in the combustion of alkane droplets, Combustion and Flame 19 (1972) 393-406.

[20] S.H. Chung, C.K. Law, An experimental study of droplet extinction in the absence of external convection, Combustion and Flame 64 (1986) 237-252. 